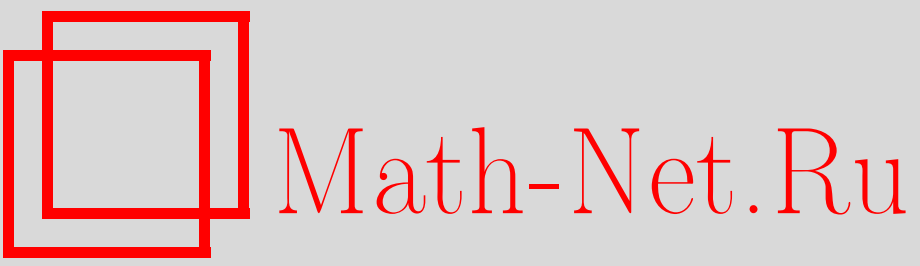

Ю. В. Павлов, Перенормировка и размерная регуляризация для скалярного поля со связью с кривизной типа Гаусса-Бонне, ТМФ, 2004, том 140, номер 2, 241255

DOI: https://doi.org/10.4213/tmf96

Использование Общероссийского математического портала Math-Net.Ru подразумевает, что вы прочитали и согласны с пользовательским соглашением http://www.mathnet.ru/rus/agreement

Параметры загрузки:

IP : 54.198 .187 .58

26 апреля 2023 г., $17: 32: 11$ 


\section{ПЕРЕНОРМИРОВКА И РАЗМЕРНАЯ РЕГУЛЯРИЗАЦИЯ ДЛЯ СКАЛЯРНОГО ПОЛЯ СО СВЯЗЬЮ С КРИВИЗНОЙ ТИПА ГАУССА-БОННЕ}

Рассматривается скалярное поле в искривленном пространстве-времени со связью с кривизной типа Гаусса-Бонне. При такой квадратичной связи с кривизной метрический тензор энергии-импульса не содержит производных от метрики выше второго порядка. Получен метрический тензор энергии-импульса. Найдена геометрическая структура первых трех контрчленов к вакуумным средним тензоров энергии-импульса для произвольной фоновой метрики $N$-мерного пространства-времени. В однородном изотропном пространстве получены первые три контрчлена $n$-волновой процедуры, позволяющие вычислять перенормированные значения вакуумных средних тензоров энергии-импульса в размерностях $N=4,5$. С помощью размерной регуляризации установлено, что геометрические структуры контрчленов в $n$-волновой процедуре и в методе эффективного действия совпадают.

Ключевые слова: скалярное поле, квантовая теория в искривленном пространстве, перенормировка, размерная регуляризация.

\section{1. ВВЕДЕНИЕ}

Квантовополевые эффекты в искривленном пространстве-времени активно исследуются в настояшее время и могут иметь важное значение в космологии ранней Вселенной и астрофизике (см. [1], [2]). При вычислениях в искривленном пространстве-времени необходимо описать взаимодействие материального поля с внешним гравитационным. Для скалярного поля рассматривались различные типы связи с кривизной. Минимальное взаимодействие заключается в замене в уравнениях свободного поля частных производных на ковариантные. Если, кроме того, в уравнение скалярного поля $\varphi(x)$ добавляется слагаемое $\xi R \varphi$, где $R$ - скалярная кривизна, то при некотором специальном выборе

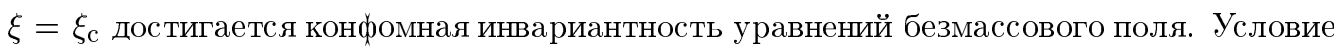
$\xi=\xi_{\mathrm{c}}$ в ряде работ выделяется как предпочтительное (см., например, [3]). Для скалярных полей в инфляционных моделях [4] обычно предполагается $\xi=0$. При исследовании квантовых эффектов в искривленном пространстве-времени активно рассматриваются модели с произвольными значениями $\xi$ (см., например, [5]). В случае взаимодействующих полей при перенормировке в искривленном пространстве невозможно сохранить

${ }^{*}$ Институт проблем машиноведения РАН и Лаборатория теоретической физики им. А. А. Фридмана, Санкт-Петербург, Россия. E-mail: pavlov@lpt.ipme.ru 
конформную инвариантность как эффективного, так и просто действия [2]. Модели с произвольным $\xi$, в которых конформная инвариантность отсутствует уже на классическом уровне, могут быть обобшены добавлением слагаемых, квадратичных по кривизне и т.д. Однако, чтобы избежать радикального изменения теории, следует сохранить важное свойство теорий со взаимодействием вида $\xi R \varphi$ : метрический тензор энергии-импульса (ТЭИ) скалярного поля с таким взаимодействием содержит производные метрики не выше второго порядка. Появление высших производных от метрики в метрическом ТЭИ, а следовательно, и в уравнениях Эйнштейна привело бы к качественному изменению теории (см. [6]) еще на классическом (!) уровне.

Требование отсутствия высших производных метрики ранее было положено в основу многомерных обобшений теории гравитации [7]. Такие условия выполняются в многомерных гравитационных теориях Эйнштейна-Гаусса-Бонне, появляющихся как низкоэнергетические приближения струнных теорий [8]. Связь скалярного поля с кривизной, при которой ТЭИ не содержит высших производных метрики, имеет место в теориях с дилатоном, связанным с кривизной посредством инварианта Гаусса-Бонне. Такие теории также появляются как низкоэнергетические следствия в струнных моделях (см., например, [9]).

В данной работе рассматривается перенормирока вакуумных средних ТЭИ скалярного поля со связью с кривизной типа Гаусса-Бонне, определяется геометрическая структура контрчленов в произвольной метрике и рассматривается $n$-волновая процедура [10] - одна из наиболее эффективных для вычисления перенормированных значений ТЭИ в однородных изотропных пространствах. В разделе 2 вычислен метрический ТЭИ и дано выражение для него, использующее конформный тензор Вейля и удобное для вычислений в однородных изотропных пространствах. В разделе 3 найдена геометрическая структура контрчленов к вакуумным средним ТЭИ в методе эффективного действия. В разделе 4 проведено квантование скалярного поля в однородном изотропном пространстве, вычислены вакуумные средние ТЭИ по вакууму, определяемому методом диагонализации гамильтониана. В разделе 5 вычислены первые три контрчлена $n$-волновой процедуры в однородном изотропном постранстве-времени, и с помошью размерной регуляризации установлено совпадение их геометрической структуры со структурой контрчленов в методе эффективного действия. В заключении суммируются результаты работы. В приложении 1 приведены выражения для вариаций некоторых геометрических величин, а также тождества Бианки и их следствия, необходимые для получения ТЭИ и контрчленов к его вакуумным средним. В приложении 2 даны выражения для встречающихся в работе геометрических величин в $N$-мерном однородном изотропном пространстве-времени.

Все вычисления проводятся для $N$-мерного пространства-времени, что необходимо для проведения размерной регуляризации и может использоваться при исследовании многомерных моделей. В статье используется система единиц, в которой $\hbar=c=1$. Знаки тензора кривизны и тензора Риччи выбраны так, что $R_{j k l}^{i}=\partial_{l} \Gamma_{j k}^{i}-\partial_{k} \Gamma_{j l}^{i}+\Gamma_{n l}^{i} \Gamma_{j k}^{n}-$ $\Gamma_{n k}^{i} \Gamma_{j l}^{n}, R_{i k}=R_{i l k}^{l}$, где $\Gamma_{j k}^{i}-$ символы Кристоффеля. 
ПЕРЕНОРМИРОВКА И РАЗМЕРНАЯ РЕГУЛЯРИЗАЦИЯ ДЛЯ СКАЛЯРНОГО ПОЛЯ . . .243

\section{2. СКАЛЯРНОЕ ПОЛЕ СО СВЯЗЬЮ С КРИВИЗНОЙ ТИПА ГАУССА-БОННЕ}

Рассмотрим комплексное скалярное поле $\varphi(x)$ массы $m$ с лагранжианом

$$
L(x)=\sqrt{|g|}\left[g^{i k} \partial_{i} \varphi^{*} \partial_{k} \varphi-\left(m^{2}+V(R)\right) \varphi^{*} \varphi\right]
$$

и соответствуюшим уравнением движения

$$
\left(\nabla^{i} \nabla_{i}+V(R)+m^{2}\right) \varphi(x)=0
$$

где $\nabla_{i}$ - ковариантные производные в $N$-мерном пространстве-времени с метрикой $g_{i k}$, $g=\operatorname{det}\left(g_{i k}\right)$; через $V(R)$ обозначена функция, зависяшая от инвариантных комбинаций тензора кривизны $R^{i}{ }_{j k l}$ и метрического тензора:

$$
V(R)=\xi R+\zeta R^{2}+\kappa R_{i j} R^{i j}+\chi R_{i j k l} R^{i j k l}+\cdots .
$$

Уравнение (2) конформно-инвариантно при $m=0$ и $V(R)=\xi_{\mathrm{c}} R$, где $\xi_{\mathrm{c}}=(N-2) /[4 \times$ $(N-1)]\left(\xi_{\mathrm{c}}=1 / 6\right.$ при $\left.N=4\right)$. Минимальной связи скалярного поля с кривизной соответствует $V(R) \equiv 0$.

Ненулевые константы при квадратичных по кривизне слагаемых в (3), имеющие размерность $[\text { масса }]^{-2}$, приводят в общем случае к наличию в метрическом ТЭИ, а следовательно и в уравнениях Эйнштейна производных метрики третьего и четвертого порядков. Как известно (см. [6]), такие добавки, даже если коэффициенты при них малы, приводят к радикальному изменению теории. Если потребовать, чтобы метрический ТЭИ скалярного поля не содержал производных метрики выше второго порядка, то в качестве $V(R)$ допустима функция

$$
V=\xi R+\zeta R_{\mathrm{GB}}^{2}
$$

где $R_{\mathrm{GB}}^{2} \stackrel{\text { def }}{=} R_{l m p q} R^{l m p q}-4 R_{l m} R^{l m}+R^{2}$. При такой связи скалярного поля с кривизной, варьируя действие по метрике, получим, используя формулы приложения 1 , следуюшее выражение для метрического ТЭИ:

$$
\begin{aligned}
T_{i k}= & \partial_{i} \varphi^{*} \partial_{k} \varphi+\partial_{k} \varphi^{*} \partial_{i} \varphi-g_{i k} \partial^{l} \varphi^{*} \partial_{l} \varphi+g_{i k} m^{2} \varphi^{*} \varphi- \\
& -2 \xi\left(G_{i k}+\nabla_{i} \nabla_{k}-g_{i k} \nabla^{l} \nabla_{l}\right)\left(\varphi^{*} \varphi\right)-2 \zeta\left(E_{i k}+P_{i k}\right)\left(\varphi^{*} \varphi\right),
\end{aligned}
$$

где $G_{i k}=R_{i k}-R g_{i k} / 2-$ тензор Эйнштейна,

$$
\begin{aligned}
E_{i k}= & \frac{\delta \int R_{\mathrm{GB}}^{2} \sqrt{|g|} d^{N} x}{\sqrt{|g|} \delta g^{i k}}=2 R_{i l m p} R_{k}^{l m p}-\frac{g_{i k}}{2} R_{l m p q} R^{l m p q}- \\
& -4 R^{l m} R_{l i m k}-4 R_{i l} R_{k}^{l}+2 g_{i k} R_{l m} R^{l m}+2 R R_{i k}-\frac{g_{i k}}{2} R^{2}, \\
P_{i k}= & 2\left[R \nabla_{i} \nabla_{k}+2 R_{i k} \nabla_{l} \nabla^{l}+2 g_{i k} R_{l m} \nabla^{l} \nabla^{m}-R g_{i k} \nabla_{l} \nabla^{l}-\right. \\
& \left.-4 R_{l(i} \nabla_{k)} \nabla^{l}-2 R_{i l k m} \nabla^{l} \nabla^{m}\right] .
\end{aligned}
$$

Здесь введено обозначение симметризации: $A_{n(i} B_{k)}=\left(A_{n i} B_{k}+A_{n k} B_{i}\right) / 2$. Отметим, что $R_{\mathrm{GB}}^{2} \equiv 0$ при $N=2,3$. Поэтому в этих размерностях не может появиться новых 
эффектов от $\zeta \neq 0$. В четырехмерном пространстве-времени $E_{i k}=0$ (см. [11]), но $P_{i k}\left(\varphi^{*} \varphi\right) \neq 0$ для метрики общего вида при $\varphi(x) \neq$ const.

В четномерных пространствах справедлива теорема Гаусса-Бонне (см., например, [12]), согласно которой для компактного ориентируемого многообразия $M$ четной размерности $N=2 k$ эйлерова характеристика, являющаяся топологическим инвариантом, равна

$$
\chi(M)=\int_{M} E(x) \sqrt{g} d^{N} x,
$$

где $g_{i k}$ - риманова метрика на $M$,

$$
E(x)=\frac{(-1)^{k}}{(4 \pi)^{k} k ! 2^{k}} \sum_{\pi, \sigma}(-1)^{\pi}(-1)^{\sigma} R_{\sigma(1) \sigma(2)}^{\pi(1) \pi(2)} \ldots R_{\sigma(N-1) \sigma(N)}^{\pi(N-1) \pi(N)} .
$$

Сумма берется по всем парам перестановок $\{\pi, \sigma\}$ набора $\{1, \ldots, N\},(-1)^{\pi},(-1)^{\sigma}$ знаки перестановок. При $N=2 \quad E(x)=-(4 \pi)^{-1} R(x)$. При $N=4$ выражение под знаком суммы в выражении (9) совпадает с $R_{\mathrm{GB}}^{2}$ (доказательство см. в работе [11]). Поэтому связь скалярного поля с кривизной в уравнении $(2)$ с $V(R)$ вида (4) естественно называть связью типа Гаусса-Бонне.

Для дальнейшего исследования ТЭИ в однородном изотропном пространстве приведем формулы для $E_{i k}$ и $P_{i k}$, выразив тензор Римана через конформный тензор Вейля:

$$
C_{i k l m}=R_{i k l m}+\frac{2}{N-2}\left(R_{m[i} g_{k] l}-R_{l[i} g_{k] m}\right)+\frac{2 R g_{l[i} g_{k] m}}{(N-1)(N-2)} .
$$

Здесь квадратные скобки в индексах обозначают антисимметризацию: $A_{n[i} B_{k] m}=$ $\left(A_{n i} B_{k m}-A_{n k} B_{i m}\right) / 2$. Подставив $(10)$ в $(6)$ и (7), получим

$$
\begin{aligned}
E_{i k}= & 2 C_{i l m p} C_{k}^{l m p}-\frac{g_{i k}}{2} C_{l m p q} C^{l m p q}-(N-4)^{(3)} H_{i k}, \\
P_{i k}= & 4\left[\frac { N - 3 } { N - 2 } \left(R_{i k} \nabla_{l} \nabla^{l}+g_{i k} R_{l m} \nabla^{l} \nabla^{m}-2 R_{l(i} \nabla_{k)} \nabla^{l}+\right.\right. \\
& \left.\left.+\frac{N R}{2(N-1)}\left(\nabla_{i} \nabla_{k}-g_{i k} \nabla_{l} \nabla^{l}\right)\right)-C_{i l k m} \nabla^{l} \nabla^{m}\right],
\end{aligned}
$$

где введенный в статье [13] тензор

$$
\begin{aligned}
{ }^{(3)} H_{i k}= & \frac{4}{N-2} C_{i l k m} R^{l m}+\frac{2(N-3)}{(N-2)^{2}}\left[2 R_{i l} R_{k}^{l}-\frac{N}{N-1} R R_{i k}-\right. \\
& \left.-g_{i k}\left(R_{l m} R^{l m}-\frac{N+2}{4(N-1)} R^{2}\right)\right]
\end{aligned}
$$

ковариантно сохраняется в конформно-плоском случае (т.е. при $C_{i k l m}=0$ ), в частности, в однородном изотропном пространстве. 
ПЕРЕНОРМИРОВКА И РАЗМЕРНАЯ РЕГУЛЯРИЗАЦИЯ ДЛЯ СКАЛЯРНОГО ПОЛЯ . . 245

\section{3. ГЕОМЕТРИЧЕСКАЯ СТРУКТУРА КОНТРЧЛЕНОВ}

В пространстве-времени с метрикой общего вида для анализа геометрической структуры расходимостей вакуумных средних ТЭИ удобно использовать размерно-регуляризованное эффективное действие. Для комплексного скалярного поля $\varphi(x)$ с уравнением движения (2) однопетлевое эффективное действие может быть записано в виде (cM. [2], [14])

$$
S_{\mathrm{eff}}=\int L_{\mathrm{eff}}(x) \sqrt{|g|} d^{N} x
$$

где

$$
\begin{gathered}
L_{\mathrm{eff}}(x)=(4 \pi)^{-N / 2}\left(\frac{M}{m}\right)^{2 \varepsilon} \sum_{j=0}^{\infty} a_{j}(x) m^{N_{0}-2 j} \Gamma\left(j-\frac{N}{2}\right), \\
a_{0}(x)=1, \quad a_{1}(x)=\frac{1}{6} R-V=\left(\frac{4-N}{12(N-1)}+\Delta \xi\right) R-\zeta R_{\mathrm{GB}}^{2}, \\
a_{2}(x)=\frac{1}{180} R_{l m p q} R^{l m p q}-\frac{1}{180} R_{l m} R^{l m}+\frac{1}{72} R^{2}-\frac{1}{30} \nabla^{l} \nabla_{l} R- \\
-\frac{1}{6} R V+\frac{1}{2} V^{2}+\frac{1}{6} \nabla^{l} \nabla_{l} V,
\end{gathered}
$$

$N$ - размерность пространства-времени, которая рассматривается как переменная, аналитически продолженная в комплексную плоскость, $\varepsilon$ - комплексный параметр, $M-$ константа размерности массы [15], введенная для сохранения обычной размерности $L_{\mathrm{eff}}$

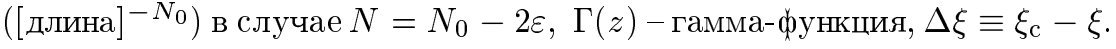

Используя (10), для связи с кривизной типа Гаусса-Бонне (4) выражение для $a_{2}$ может быть записано следующим образом:

$$
\begin{aligned}
a_{2}(x)= & \frac{(N-6) R_{\mathrm{GB}}^{2}}{720(N-3)}+\frac{(N-2) C_{l m p q} C^{l m p q}}{240(N-3)}-\frac{1}{6} \nabla^{l} \nabla_{l}\left(\frac{1}{5} R-V\right)+ \\
& +\left(\frac{(N-4)(N-6)}{480(N-1)^{2}}-\frac{\Delta \xi(N-4)}{12(N-1)}+\frac{(\Delta \xi)^{2}}{2}\right) R^{2}+ \\
& +\left(\frac{(N-4)}{12(N-1)}-\Delta \xi\right) \zeta R R_{\mathrm{GB}}^{2}+\frac{1}{2} \zeta^{2} R_{\mathrm{GB}}^{4} .
\end{aligned}
$$

Первые $\left[N_{0} / 2\right]+1$ слагаемых в (15) исключаются для получения перенормированного $L_{\text {eff }}([b]$ обозначает целую часть числа $b)$. Варьированием по $g_{i k}$ соответствующих $j=0,1,2$ слагаемых в эффективном действии получим, с использованием формул приложения 1 , вычитаемые из вакуумного ТЭИ члены

$$
\begin{aligned}
T_{i k, \varepsilon}[0]= & -\frac{m^{N_{0}}}{2^{N_{0}} \pi^{N_{0} / 2}}\left(\frac{4 \pi M^{2}}{m^{2}}\right)^{\varepsilon} \Gamma\left(\varepsilon-\frac{N_{0}}{2}\right) g_{i k} \\
T_{i k, \varepsilon}[1]= & \frac{m^{N_{0}-2}}{2^{N_{0}-1} \pi^{N_{0} / 2}}\left(\frac{4 \pi M^{2}}{m^{2}}\right)^{\varepsilon} \Gamma\left(1-\frac{N}{2}\right)\left[\left(\frac{1}{6}-\xi\right) G_{i k}-\zeta E_{i k}\right]= \\
= & \frac{m^{N_{0}-2}}{2^{N_{0}-1} \pi^{N_{0} / 2}}\left(\frac{4 \pi M^{2}}{m^{2}}\right)^{\varepsilon}\left[\Delta \xi \Gamma\left(1-\frac{N}{2}\right) G_{i k}-\right. \\
& \left.-\frac{\Gamma(3-N / 2)}{(N-2)}\left(\frac{G_{i k}}{3(N-1)}+\zeta \frac{4 E_{i k}}{(N-4)}\right)\right]
\end{aligned}
$$




$$
\begin{aligned}
T_{i k, \varepsilon}[2]= & \frac{m^{N_{0}-4}}{(4 \pi)^{N_{0} / 2}}\left(\frac{4 \pi M^{2}}{m^{2}}\right)^{\varepsilon}\left\{\frac{\Gamma(2-N / 2)}{360(N-3)}\left[(N-6) E_{i k}+3(N-2) W_{i k}\right]+\right. \\
& +\left[\frac{\Gamma(4-N / 2)}{60(N-1)^{2}}+\Delta \xi \frac{\Gamma(3-N / 2)}{3(N-1)}+(\Delta \xi)^{2} \Gamma\left(2-\frac{N}{2}\right)\right]{ }^{(1)} H_{i k}- \\
& -\zeta \frac{\Gamma(3-N / 2)}{3(N-1)}\left[\left(R_{i k}+\nabla_{i} \nabla_{k}-g_{i k} \nabla_{l} \nabla^{l}\right) R_{\mathrm{GB}}^{2}+\left(E_{i k}+P_{i k}\right) R\right]+ \\
& +\zeta 2 \Gamma\left(2-\frac{N}{2}\right)\left[\zeta\left(\frac{g_{i k}}{4} R_{\mathrm{GB}}^{2}+E_{i k}+P_{i k}\right) R_{\mathrm{GB}}^{2}-\right. \\
& \left.\left.-\Delta \xi\left(\left(R_{i k}+\nabla_{i} \nabla_{k}-g_{i k} \nabla_{l} \nabla^{l}\right) R_{\mathrm{GB}}^{2}+\left(E_{i k}+P_{i k}\right) R\right)\right]\right\}
\end{aligned}
$$

где

$$
\begin{aligned}
{ }^{(1)} H_{i k}= & \frac{\delta \int R^{2} \sqrt{|g|} d^{N} x}{\sqrt{|g|} \delta g^{i k}}=2\left(\nabla_{i} \nabla_{k} R-g_{i k} \nabla^{l} \nabla_{l} R\right)+2 R\left(R_{i k}-\frac{1}{4} R g_{i k}\right) \\
W_{i k}= & \frac{\delta \int C_{l m p q} C^{l m p q} \sqrt{|g|} d^{N} x}{\sqrt{|g|} \delta g^{i k}}= \\
= & E_{i k}+\frac{4(N-3)}{N-2}\left(2 R^{l m} R_{l i m k}-\frac{g_{i k}}{2} R_{l m} R^{l m}-\frac{N R R_{i k}}{2(N-1)}+\frac{N R^{2} g_{i k}}{8(N-1)}+\right. \\
& \left.+\frac{N-2}{2(N-1)} \nabla_{i} \nabla_{k} R+\frac{g_{i k}}{2(N-1)} \nabla^{l} \nabla_{l} R-\nabla^{l} \nabla_{l} R_{i k}\right) .
\end{aligned}
$$

В конформно-плоском случае $C_{i k l m}=0, E_{i k} /(4-N)={ }^{(3)} H_{i k}, W_{i k}=0$ и, следовательно,

$$
\begin{aligned}
T_{i k, \varepsilon}[1]= & \frac{m^{N_{0}-2}}{2^{N_{0}-1} \pi^{N_{0} / 2}}\left(\frac{4 \pi M^{2}}{m^{2}}\right)^{\varepsilon}\left[\Delta \xi \Gamma\left(1-\frac{N}{2}\right) G_{i k}+\right. \\
& \left.+\frac{\Gamma(3-N / 2)}{(N-2)}\left(\zeta 4^{(3)} H_{i k}-\frac{G_{i k}}{3(N-1)}\right)\right] \\
T_{i k, \varepsilon}[2]= & \frac{m^{N_{0}-4}}{(4 \pi)^{N_{0} / 2}}\left(\frac{4 \pi M^{2}}{m^{2}}\right)^{\varepsilon}\left\{{ } ^ { ( 3 ) } H _ { i k } \left[\frac{-\Gamma(4-N / 2)}{90(N-3)}+\right.\right. \\
& \left.+\zeta 4 \Gamma\left(3-\frac{N}{2}\right)\left(\left(\frac{N-4}{12(N-1)}-\Delta \xi\right) R+\zeta R_{\mathrm{GB}}^{2}\right)\right]+ \\
& +H_{i k}^{(1)}\left[\frac{\Gamma(4-N / 2)}{60(N-1)^{2}}+\Delta \xi \frac{\Gamma(3-N / 2)}{3(N-1)}+(\Delta \xi)^{2} \Gamma\left(2-\frac{N}{2}\right)\right]- \\
& -\zeta \frac{\Gamma(3-N / 2)}{3(N-1)}\left[\left(R_{i k}+\nabla_{i} \nabla_{k}-g_{i k} \nabla_{l} \nabla^{l}\right) R_{\mathrm{GB}}^{2}+P_{i k} R\right]+ \\
& +\zeta 2 \Gamma\left(2-\frac{N}{2}\right)\left[\zeta\left(\frac{g_{i k}}{4} R_{\mathrm{GB}}^{2}+P_{i k}\right) R_{\mathrm{GB}}^{2}-\right. \\
& \left.\left.-\Delta \xi\left(\left(R_{i k}+\nabla_{i} \nabla_{k}-g_{i k} \nabla_{l} \nabla^{l}\right) R_{\mathrm{GB}}^{2}+P_{i k} R\right)\right]\right\} .
\end{aligned}
$$


В предположении, что вакуумные средние ТЭИ $\left\langle T_{i k}\right\rangle$ являются источниками гравитационного поля (см. [1], [2]), т.е.

$$
G_{i k}+\Lambda g_{i k}=-8 \pi G\left(T_{i k}^{b}+\left\langle T_{i k}\right\rangle\right)
$$

где $\Lambda, G$ - космологическая и гравитационная постоянные, $T_{i k}^{b}-$ ТЭИ фоновой материи, формулы (19)-(21) позволяют сделать следуюший вывод. Первые три вычитания из вакуумного ТЭИ в $N$-мерном пространстве-времени соответствуют перенормировке космологической и гравитационной постоянных и параметров при квадратичных, кубических и четвертой степени по кривизне слагаемых в затравочном гравитационном лагранжиане следуюшего вида:

$$
L_{g r, \varepsilon}=\sqrt{|g|}\left[\frac{R-2 \Lambda_{\varepsilon}}{16 \pi G_{\varepsilon}}+\alpha_{\varepsilon} R_{\mathrm{GB}}^{2}+\beta_{\varepsilon} R^{2}+\gamma_{\varepsilon} C_{l m p q} C^{l m p q}+\delta_{\varepsilon} R R_{\mathrm{GB}}^{2}+\theta_{\varepsilon} R_{\mathrm{GB}}^{4}\right] .
$$

Вычитание $T_{i k, \varepsilon}[0]$ согласно (19) соответствует бесконечной перенормировке космологической постоянной $\Lambda_{\varepsilon}$. Из (20) следует, что вычитание $T_{i k, \varepsilon}[1]$ соответствует перенормировке гравитационной постоянной $G_{\varepsilon}$ (конечной при $N \rightarrow 4$ для $\xi=\xi_{\text {c }}$ ) и бесконечной перенормировке параметра $\alpha_{\varepsilon}$ (при $\left.\zeta \neq 0\right)$. Вычитание $T_{i k, \varepsilon}[2]$ согласно (18) и (21) соответствует перенормировке параметров $\alpha_{\varepsilon}, \beta_{\varepsilon}, \gamma_{\varepsilon}, \delta_{\varepsilon}, \theta_{\varepsilon}$. При $\xi=\xi_{\mathrm{c}}$ и $N \rightarrow 4$ параметры $\beta_{\varepsilon}$ и $\delta_{\varepsilon}$ испытывают конечную перенормировку.

Отметим, что запись в (27) слагаемого с $\alpha_{\varepsilon} R_{\mathrm{GB}}^{2}$ согласована с использованием размерной регуляризации $\left(E_{i k} \equiv 0\right.$ только при целых $\left.N=2,3,4\right)$.

При $N \rightarrow 4$ произведения $E_{i k} \Gamma(1-(N / 2))$ и $E_{i k} \Gamma(2-(N / 2))$ имеют конечные пределы для произвольной метрики пространства-времени, поскольку при аналитическом продолжении по размерности зависимость выражений для $E_{i k}$ от $N$ предполагается дробно-рациональной, $E_{i k}=0$ при $N=4$ и гамма-функция имеет в точках 0 и -1 полюсы первого порядка. Поэтому соответствуюшие слагаемые в (20) и (21) конечны, и для получения конечных величин в уравнении (26), описываюшем обратное влияние квантованного поля на метрику, их выгитание не является необходимым. Однако без таких вычитаний эффективное действие останется расходящимся, и выражение для аномального следа вакуумного ТЭИ будет отличаться от стандартного даже при $\zeta=0$. Поскольку соответствуюшие слагаемые появляются при использовании различных методов регуляризации (см. [1], [2]), обшепринято сохранять их в контрчленах к вакуумному ТЭИ.

Вопросы о необходимости конечных перенормировок и о значениях ренормированных параметров относятся к области эксперимента. Возможно, как отмечено в [1], что ренормированные параметры при неэйнштейновских слагаемых в гравитационном лагранжиане равны нулю.

\section{4. СКАЛЯРНОЕ ПОЛЕ В ОДНОРОДНОМ ИЗОТРОПНОМ ПРОСТРАНСТВЕ}

Метрику $N$-мерного однородного изотропного пространства-времени запишем в виде

$$
d s^{2}=g_{i k} d x^{i} d x^{k}=a^{2}(\eta)\left(d \eta^{2}-d l^{2}\right),
$$


где $d l^{2}=\gamma_{\alpha \beta} d x^{\alpha} d x^{\beta}$ - метрика $(N-1)$-мерного пространства постоянной кривизны $K=0, \pm 1$. Полная система решений уравнения (2) в метрике (28) может быть найдена в форме

$$
\varphi(x)=a^{-(N-2) / 2}(\eta) g_{\lambda}(\eta) \Phi_{J}(\mathbf{x})
$$

где

$$
\begin{gathered}
g_{\lambda}^{\prime \prime}(\eta)+\Omega^{2}(\eta) g_{\lambda}(\eta)=0 \\
\Omega^{2}(\eta)=m^{2} a^{2}+\lambda^{2}-\Delta \xi a^{2} R+\zeta a^{2} R_{\mathrm{GB}}^{2} \\
\Delta_{N-1} \Phi_{J}(\mathbf{x})=-\left(\lambda^{2}-\left(\frac{N-2}{2}\right)^{2} K\right) \Phi_{J}(\mathbf{x})
\end{gathered}
$$

штрих обозначает производную по конформному времени $\eta, J$ - набор индексов (квантовых чисел), нумерующих собственные функции оператора Лапласа-Бельтрами $\Delta_{N-1}$ в $(N-1)$-мерном пространстве. Отметим, что из неотрицательности собственных значений оператора $-\Delta_{N-1}$ следует неравенство $\lambda^{2}-((N-2) / 2)^{2} K \geqslant 0$.

В соответствии с методом диагонализации гамильтониана [1] (случай произвольной $V(R)$ cм. в работе [16]), функции $g_{\lambda}(\eta)$ должны удовлетворять следуюшим начальным условиям:

$$
g_{\lambda}^{\prime}\left(\eta_{0}\right)=i \Omega\left(\eta_{0}\right) g_{\lambda}\left(\eta_{0}\right), \quad\left|g_{\lambda}\left(\eta_{0}\right)\right|=\Omega^{-1 / 2}\left(\eta_{0}\right) .
$$

Для квантования разложим поле $\varphi(x)$ по полной системе решений $(29)$ :

$$
\varphi(x)=\int d \mu(J)\left[\varphi_{J}^{(+)} a_{J}^{(+)}+\varphi_{J}^{(-)} a_{J}^{(-)}\right]
$$

где

$$
\varphi_{J}^{(+)}(x)=\frac{a^{-(N-2) / 2}(\eta)}{\sqrt{2}} g_{\lambda}(\eta) \Phi_{J}^{*}(\mathbf{x}), \quad \varphi_{J}^{(-)}(x)=\left(\varphi_{J}^{(+)}(\mathbf{x})\right)^{*},
$$

и потребуем выполнения коммутационных соотношений

$$
\left[a_{J}^{(-)}, \stackrel{*}{a} \underset{J^{\prime}}{(+)}\right]=\left[\stackrel{*}{a}_{J}^{(-)}, a_{J^{\prime}}^{(+)}\right]=\delta_{J J^{\prime}}, \quad\left[a_{J}^{( \pm)}, a_{J^{\prime}}^{( \pm)}\right]=\left[a_{J}^{*}( \pm), \stackrel{*}{a} \underset{J^{\prime}}{( \pm)}\right]=0 .
$$

Средние от оператора ТЭИ по вакууму $|0\rangle$, уничтожаемому операторами $a_{J}^{(-)}, \stackrel{*(-)}{a_{J^{\prime}}}$, удобно выражать через билинейные комбинации функций $g_{\lambda}$ и $g_{\lambda}^{*}$ :

$$
S=\frac{\left|g_{\lambda}^{\prime}\right|^{2}+\Omega^{2}\left|g_{\lambda}\right|^{2}}{4 \Omega}-\frac{1}{2}, \quad U=\frac{\Omega^{2}\left|g_{\lambda}\right|^{2}-\left|g_{\lambda}^{\prime}\right|^{2}}{2 \Omega}, \quad V=-\frac{d\left(g_{\lambda}^{*} g_{\lambda}\right)}{2 d \eta},
$$

которые в соответствии с (30) удовлетворяют системе дифференциальных уравнений

$$
S^{\prime}=\frac{\Omega^{\prime}}{2 \Omega} U, \quad U^{\prime}=\frac{\Omega^{\prime}}{\Omega}(1+2 S)-2 \Omega V, \quad V^{\prime}=2 \Omega U .
$$

С учетом начальных условий $S\left(\eta_{0}\right)=U\left(\eta_{0}\right)=V\left(\eta_{0}\right)=0$, следуюших из (33), уравнения (38) могут быть записаны в виде системы интегральных уравнений Вольтерра:

$$
\begin{gathered}
U(\eta)+i V(\eta)=\int_{\eta_{0}}^{\eta} w\left(\eta_{1}\right)\left(1+2 S\left(\eta_{1}\right)\right) e^{2 i \Theta\left(\eta_{1}, \eta\right)} d \eta_{1}, \\
S(\eta)=\frac{1}{2} \int_{\eta_{0}}^{\eta} d \eta_{1} w\left(\eta_{1}\right) \int_{\eta_{0}}^{\eta_{1}} d \eta_{2} w\left(\eta_{2}\right)\left(1+2 S\left(\eta_{2}\right)\right) \cos \left[2 \Theta\left(\eta_{2}, \eta_{1}\right)\right],
\end{gathered}
$$



где $w(\eta)=\Omega^{\prime}(\eta) / \Omega(\eta), \Theta\left(\eta_{1}, \eta_{2}\right)=\int_{\eta_{1}}^{\eta_{2}} \Omega(\eta) d \eta$.

Для получения вакуумного ТЭИ используем следуюшие формулы суммирования для собственных функций оператора $\Delta_{N-1}$ (см. [17]):

$$
\begin{gathered}
\sum_{J(\lambda=\text { const })}\left|\Phi_{J}(\mathbf{x})\right|^{2}=f(\lambda), \\
\sum_{J(\lambda=\text { const })}\left[\left(\partial_{\alpha} \Phi_{J}^{*}\right) \partial_{\beta} \Phi_{J}+\left(\partial_{\beta} \Phi_{J}^{*}\right) \partial_{\alpha} \Phi_{J}\right]=\frac{2 \gamma_{\alpha \beta}}{N-1}\left(\lambda^{2}-\left(\frac{N-2}{2}\right)^{2} K\right) f(\lambda), \\
\sum_{J(\lambda=\text { const })}\left[\left(\widetilde{\nabla}_{\alpha} \widetilde{\nabla}_{\beta} \Phi_{J}^{*}\right) \Phi_{J}+\Phi_{J}^{*} \widetilde{\nabla}_{\alpha} \widetilde{\nabla}_{\beta} \Phi_{J}\right]=-\frac{2 \gamma_{\alpha \beta}}{N-1}\left(\lambda^{2}-\left(\frac{N-2}{2}\right)^{2} K\right) f(\lambda),
\end{gathered}
$$

где знак $\sum$ для непрерывного $J$ обозначает интегрирование, $\widetilde{\nabla}_{\alpha}-$ ковариантное дифференцирование в $(N-1)$-мерном пространстве с метрикой $\gamma_{\alpha \beta}$. В квазиевклидовом $(K=0)$ случае функция $f(\lambda)$ равна

$$
f(\lambda)=\frac{B_{N}}{2} \lambda^{N-2}, \quad B_{N}=\left[2^{N-3} \pi^{(N-1) / 2} \Gamma\left(\frac{N-1}{2}\right)\right]^{-1}
$$

(случай $K \neq 0$ см. в работе [17]).

Подставив разложение (34) в (5) и используя (11)-(13), (36), (37), (41)-(43) и формулы приложения 2, для вакуумных средних ТЭИ получим следуюшие (расходяшиеся) выражения:

$$
\left\langle 0\left|T_{i k}\right| 0\right\rangle=\frac{B_{N}}{a^{N-2}} \int d \mu(\lambda) \tau_{i k},
$$

где

$$
\begin{aligned}
\tau_{00}= & \Omega\left(S+\frac{1}{2}\right)+(N-1)\left(\Delta \xi-\tilde{\zeta}\left(c^{2}+K\right)\right) \times \\
& \times\left[c V+\left(c^{\prime}+(N-2) c^{2}\right) \frac{1}{\Omega}\left(S+\frac{1}{2} U+\frac{1}{2}\right)\right], \\
\tau_{\alpha \beta}= & \gamma_{\alpha \beta}\left\{\frac{1}{(N-1) \Omega}\left[\lambda^{2}\left(S+\frac{1}{2}\right)-\left(\Omega^{2}-\lambda^{2}\right) \frac{U}{2}\right]-\right. \\
& -2\left(\Delta \xi-\tilde{\zeta}\left(c^{2}+K\right)\right) \Omega U+\left[\Delta \xi(N-1)-\tilde{\zeta}\left((N+1)\left(c^{2}+K\right)-2 c^{\prime}\right)\right] c V- \\
& -\frac{1}{\Omega}\left(S+\frac{1}{2} U+\frac{1}{2}\right)\left[\Delta \xi\left((N-1) c^{\prime}+(N-2) K\right)-\right. \\
& \left.\left.-\tilde{\zeta}\left((N-1) c^{2}\left(3 c^{\prime}-2\left(c^{2}+K\right)\right)+K\left((N+1) c^{\prime}+(N-4)\left(c^{2}+K\right)\right)\right)\right]\right\},
\end{aligned}
$$

$\tilde{\zeta} \equiv \zeta a^{-2} 2(N-2)(N-3), c \equiv a^{\prime} / a$.

В четырехмерном пространстве-времени мера интегрирования в выражении (45) имеет вид (см. [1])

$$
\int d \mu(\lambda) \ldots= \begin{cases}\int_{0}^{\infty} d \lambda \lambda^{2} \ldots, & K=0,-1 \\ \sum_{\lambda=1}^{\infty} \lambda^{2} \ldots, & K=1\end{cases}
$$


В $N$-мерном случае $d \mu(\lambda)=\sigma(\lambda) d \lambda($ см. [17]), где

$$
\begin{aligned}
\sigma(\lambda) & =\lambda^{N-2}+\alpha_{N} \lambda^{N-4}+\beta_{N} \lambda^{N-6}+\cdots \\
\alpha_{N} & =-\frac{1}{24}(N-2)(N-3)(N-4) K \\
\beta_{N} & =\frac{1}{5760}(N-2)(N-3)(N-4)(N-5)(N-6)(5 N-8) K^{2} .
\end{aligned}
$$

\section{5. n-ВОЛНОВАЯ ПРОЦЕДУРА}

В однородных изотропных пространствах для вычисления перенормированого вакуумного ТЭИ часто применяется $n$-волновая процедура, предложенная в работе [10]. Для $N$-мерного однородного изотропного пространства-времени $n$-волновую процедуру можно выразить следуюшими формулами (см. [17]):

$$
\left\langle 0\left|T_{i k}\right| 0\right\rangle_{\mathrm{ren}}=\frac{B_{N}}{a^{N-2}} \lim _{\Lambda \rightarrow \infty}\left[\int^{\Lambda} d \mu(\lambda) \tau_{i k}-\sum_{l=0}^{[N / 2]} \int_{0}^{\Lambda} d \lambda \lambda^{N-2} a_{i k}[l]\right],
$$

где

$$
a_{i k}[l]=\frac{1}{l !} \lim _{n \rightarrow \infty} \frac{\partial^{l}}{\partial\left(n^{-2}\right)^{l}}\left(\frac{\tau_{i k}(n \lambda, n m) \sigma(n \lambda)}{n^{N-1} \lambda^{N-2}}\right) .
$$

Для получения явного вида $a_{i k}[l]$ разложим $S, U$ и $V$ (см. (37)) по обратным степеням $n$ при замене $\lambda \rightarrow n \lambda, m \rightarrow n m$ и $n \rightarrow \infty: S=\sum_{k=1}^{\infty} n^{-k} S_{k}, \ldots$ Используя последовательные итерации в интегральных уравнениях $(39),(40)$ и метод стационарной фазы, получим для первых отличных от нуля членов разложений

$$
\begin{gathered}
V_{1}=W, \quad U_{2}=D W, \quad S_{2}=\frac{1}{4} W^{2}, \quad V_{3}=\frac{1}{2} W^{3}-D^{2} W-\frac{\omega}{2} D\left(\frac{q}{\omega^{3}}\right), \\
U_{4}=\frac{3}{2} W^{2} D W-D^{3} W-D\left(\frac{\omega}{2} D\left(\frac{q}{\omega^{3}}\right)\right)+\frac{q}{2 \omega^{2}} D W \\
S_{4}=\frac{3}{16} W^{4}+\frac{1}{4}(D W)^{2}-\frac{1}{2} W D^{2} W-\frac{1}{4} \omega W D\left(\frac{q}{\omega^{3}}\right)
\end{gathered}
$$

где

$$
q=\left(\Delta \xi R-\zeta R_{\mathrm{GB}}^{2}\right) a^{2}, \quad \omega=\left(m^{2} a^{2}+\lambda^{2}\right)^{1 / 2}, \quad W=\frac{\omega^{\prime}}{2 \omega^{2}}, \quad D=\frac{1}{2 \omega} \frac{d}{d \eta} .
$$

Отметим, что в выражения (54)-(56) исключены нелокальные по времени (т.е. зависящие как от $\eta$, так и от $\left.\eta_{0}\right)$ слагаемые. Такие слагаемые отсутствуют, если $V_{1}\left(\eta_{0}\right)=$ $V_{3}\left(\eta_{0}\right)=U_{2}\left(\eta_{0}\right)=U_{4}\left(\eta_{0}\right)=0$, что и предполагается далее. В частности, нелокальные слагаемые отсутствуют, когда первые $2[N / 2]$ производных от масштабного фактора $a(\eta)$ метрики обрашаются в нуль в начальный момент времени. 
Используя (46), (47), (49), (54)-(56), для $a_{i k}[l]$ получим

$$
\begin{array}{llrl}
a_{00}[0] & =\tau_{00}[0]=\frac{\omega}{2}, & a_{\alpha \beta}[0] & =\tau_{\alpha \beta}[0]=\gamma_{\alpha \beta} \frac{\lambda^{2}}{2(N-1) \omega}, \\
a_{i k}[1] & =\tau_{i k}[1]+\frac{\alpha_{N}}{\lambda^{2}} \tau_{i k}[0], & a_{i k}[2] & =\tau_{i k}[2]+\frac{\alpha_{N}}{\lambda^{2}} \tau_{i k}[1]+\frac{\beta_{N}}{\lambda^{4}} \tau_{i k}[0],
\end{array}
$$

где

$$
\begin{aligned}
\tau_{00}[1]= & \omega S_{2}+(N-1)\left(\Delta \xi-\tilde{\zeta}\left(c^{2}+K\right)\right) c V_{1}+ \\
& +\frac{N-1}{8 \omega}\left[\Delta \xi 2(N-2)\left(c^{2}-K\right)+\tilde{\zeta}\left(c^{2}+K\right)\left((4-3 N) c^{2}+(N-4) K\right)\right] \\
\tau_{\alpha \beta}[1]= & \gamma_{\alpha \beta}\left\{\frac{1}{(N-1) \omega}\left[\lambda^{2} S_{2}-\frac{m^{2} a^{2}}{2}\left(U_{2}+\frac{q}{2 \omega^{2}}\right)\right]-2 \Delta \xi \omega U_{2}+\right. \\
& +2 \tilde{\zeta}\left(c^{2}+K\right) \omega U_{2}+\left[\Delta \xi(N-1)-\tilde{\zeta}\left((N+1)\left(c^{2}+K\right)-2 c^{\prime}\right)\right] c V_{1}+ \\
& +\frac{1}{4 \omega}\left[\Delta \xi(N-2)\left(c^{2}-K-2 c^{\prime}\right)+\tilde{\zeta}\left(c^{2}(3 N-4)\left(2 c^{\prime}-\frac{3}{2} c^{2}\right)+\right.\right. \\
& \left.\left.\left.+K\left(2 N c^{\prime}-3 N c^{2}+\frac{3}{2}(N-4) K\right)\right)\right]\right\} \\
\tau_{00}[2]= & \omega\left(S_{4}+\frac{q}{4 \omega^{2}} U_{2}+\frac{q^{2}}{16 \omega^{4}}\right)+(N-1)\left(\Delta \xi-\tilde{\zeta}\left(c^{2}+K\right)\right) c V_{3}+ \\
& +\left[\Delta \xi 2(N-2)\left(c^{2}-K\right)+\tilde{\zeta}\left(c^{2}+K\right)\left((N-4) K-(3 N-4) c^{2}\right)\right] \times \\
& \times \frac{N-1}{4 \omega}\left(S_{2}+\frac{1}{2} U_{2}+\frac{q}{4 \omega^{2}}\right) \\
\tau_{\alpha \beta}[2]= & \gamma_{\alpha \beta}\left\{\frac{1}{N-1}\left[\frac{\lambda^{2}}{\omega}\left(S_{4}+\frac{q U_{2}}{4 \omega^{2}}+\frac{q^{2}}{16 \omega^{4}}\right)-\frac{m^{2} a^{2}}{2 \omega}\left(U_{4}+\frac{q^{2}}{4 \omega^{4}}+\frac{q S_{2}}{\omega^{2}}\right)\right]+\right. \\
& +\left[\Delta \xi(N-1)-\tilde{\zeta}\left((N+1)\left(c^{2}+K\right)-2 c^{\prime}\right)\right] c V_{3}- \\
& -2\left(\Delta \xi-\tilde{\zeta}\left(c^{2}+K\right)\right)\left(\omega U_{4}-\frac{q U_{2}}{2 \omega}\right)+ \\
& +\left[\Delta \xi(N-2)\left(c^{2}-K-2 c^{\prime}\right)+\tilde{\zeta}\left(c^{2}(3 N-4)\left(2 c^{\prime}-\frac{3}{2} c^{2}\right)+\right.\right. \\
& \left.\left.\left.+K\left(2 N c^{\prime}-3 N c^{2}+\frac{3}{2}(N-4) K\right)\right)\right] \frac{1}{2 \omega}\left(S_{2}+\frac{1}{2} U_{2}+\frac{q}{4 \omega^{2}}\right)\right\} \\
& {[(63)) } \\
& {[(61)) } \\
& {[(2)) } \\
&
\end{aligned}
$$

Приведенные выражения исчерпывают все вычитания в размерностях $N=4,5$.

Перенормированный в соответствии с (52) вакуумный ТЭИ ковариантно сохраняется. Это доказывается на основании выражений (58), (59) и равенств $\nabla^{i}\left(\tau_{i k} / a^{N-2}\right)=0$ и $\nabla^{i}\left(\tau_{i k}[l] / a^{N-2}\right)=0$, которые следуют из $(38),(46),(47),(60)-(63)$. 
Для выяснения геометрической структуры контрчленов $n$-волновой процедуры проведем, как и в работе [18], размерную регуляризацию. При вычислении интегралов в размерно-регуляризованных контрчленах

$$
T_{i k, \varepsilon}[l]=\frac{B_{N}}{a^{N-2}}(M)^{2 \varepsilon} \int_{0}^{\infty} d \lambda \lambda^{N-2} a_{i k, \varepsilon}[l]
$$

где $a_{i k, \varepsilon}[l]$ определяются по формулам (58)-(63) с заменой $N \rightarrow N_{0}-2 \varepsilon$, используем равенство

$$
\int_{0}^{\infty} x^{k}\left(1+x^{2}\right)^{-p} d x=\frac{\Gamma((k+1) / 2) \Gamma(p-(k+1) / 2)}{2 \Gamma(p)} .
$$

В тех случаях, когда интеграл в левой части формулы (65) не сушествует в обычном смысле, он полагается равным аналитическому продолжению правой части формулы (65) на соответствующие значения $k$ и $p$. В результате громоздких, но не содержащих принципиальных трудностей вычислений с учетом формул приложения 2 получим для 0-го, 1-го и 2-го контрчленов $n$-волновой процедуры выражения (19), (24), (25), соответственно. Следовательно, геометрическая структура первых трех вычитаний в $n$-волновой процедуре и в методе эффективного действия совпадают.

\section{6. ЗАКЛЮЧЕНИЕ}

В данной работе рассмотрено скалярное поле со связью с кривизной типа ГауссаБонне, при которой метрический ТЭИ не содержит производных от метрики выше второго порядка. Получены выражения для ТЭИ (5)-(7) и дано их представление через конформный тензор Вейля, удобное для вычислений как в конформно-плоских (в частности, в однородных изотропных) пространствах, так и в Риччи-плоских пространствах (т.е. при $R_{i k}=0$; см. $(11),(12)$ ). Найдена геометрическая структура первых трех контрчленов к вакуумному ТЭИ в произвольной метрике для $N$-мерного пространства-времени (см. (19)-(21)). В размерностях $N=4,5$ эти контрчлены исчерпывают все вычитания. Анализ геометрической структуры этих контрчленов позволяет сделать вывод, что первые три вычитания соответствуют перенормировке космологической и гравитационной постоянных и параметров при квадратичных, кубических и четвертой степени по кривизне слагаемых в затравочном гравитационном лагранжиане вида (27). Для однородного изотропного пространства-времени получены формулы (45)-(47), определяющие неперенормированные вакуумные средние ТЭИ. Найдены первые три контрчлена $n$-волновой процедуры (52) для вакуумного ТЭИ (см. (58)-(63)). С помощью размерной регуляризации установлено, что геометрическая структура контрчленов $n$-волновой процедуры в однородном изотропном пространстве совпадает со структурой контрчленов, полученных в методе эффективного действия (19), (24), (25). Формулы (45)-(47), (52), (58)-(63) позволяют вычислять перенормированные вакуумные средние ТЭИ скалярного поля со связью с кривизной типа Гаусса-Бонне в однородном изотропном пространстве-времени в размерностях $N=4,5$.

Учет возможной связи скалярного поля с инвариантом Гаусса-Бонне $R_{\mathrm{GB}}^{2}$ может играть важную роль в ранней Вселенной. Эффекты от ненулевого значения параметра $\zeta$ в уравнениях скалярного поля могут проявляться в излучении черных дыр (случай дилатона, связанного с инвариантом Гаусса-Бонне, см. в работе [19]), влиять на параметры 
так называемых бозонных звезд (см., например, работу [20]) и т.д. Вопросы о величине параметра $\zeta$, так же как и параметра $\xi$, относятся в конечном итоге к области эксперимента.

\section{ПРИЛОЖЕНИЕ А}

Здесь приведены выражения для вариаций некоторых геометрических величин, а также тождества Бианки и их следствия, необходимые для получения ТЭИ скалярного поля со связью с кривизной типа Гаусса-Бонне и контрчленов к его вакуумным средним. Точки с запятой в индексах обозначают соответствуюшие ковариантные производные.

При вычислении вариаций обозначим вариации метрик $\delta g_{i k}=h_{i k}$ и $h=h_{i}^{i}$. Тогда $\delta g^{i k}=-h^{i k}$,

$$
\begin{aligned}
& \delta \sqrt{|g|}=-\frac{1}{2} \sqrt{|g|} g_{i k} \delta g^{i k}=\frac{1}{2} \sqrt{|g|} h, \quad \delta \Gamma_{k l}^{i}=\frac{1}{2}\left(h_{k ; l}^{i}+h_{l ; k}^{i}-h_{k l}{ }^{; i}\right), \\
& \delta R_{j k l}^{i}=\frac{1}{2}\left(h_{j ; k l}^{i}+h_{k ; j l}^{i}-h_{j k}{ }^{; i}{ }_{l}-h_{l ; j k}^{i}-h_{j ; l k}^{i}+h_{j l} ;{ }_{k}\right), \\
& \delta R_{i k}=\frac{1}{2}\left(h_{; i k}+h_{i k}{ }^{l} l-h_{i ; k l}^{l}-h_{k ; i l}^{l}\right), \\
& \delta R=h_{; l} l-h_{l m} ; l m-R^{l m} h_{l m}, \\
& \delta\left(R^{2}\right)=2 R h_{; l}^{l}-2 R h_{l m} ; l m-2 R R^{l m} h_{l m}, \\
& \delta\left(R_{i k} R^{i k}\right)=R^{i k}\left(h_{; i k}+h_{i k}{ }^{; l}{ }_{l}-h_{i ; k l}^{l}-h_{k ; i l}^{l}\right)-2 R_{i l} R_{k}^{l} h^{i k}, \\
& \delta\left(R_{l m p q} R^{l m p q}\right)=2 R^{l m p q}\left(h_{l p ; m q}+h_{l p ; q m}\right)-2 R_{l m p q} R_{i}{ }^{m p q} h^{l i}, \\
& \delta\left(R_{l m p q} R^{l m p q}-4 R_{l m} R^{l m}+R^{2}\right)= \\
& =2\left[\left(4 R_{i l} R_{k}^{l}-R_{i l p q} R_{k}{ }^{l p q}-R R_{i k}\right) h^{i k}+R_{i{ }_{k}{ }^{q}\left(h^{i k} ; l q\right.}+h^{i k}{ }_{; q l}\right)+ \\
& \left.+R h_{; l}^{l}-R h_{; i k}^{i k}-2 R^{l m} h_{; l m}-2 R_{i k} h_{; l}^{i k}{ }^{l}+4 R_{i l} h^{i k ; l}{ }_{k}\right] .
\end{aligned}
$$

Тождества Бианки для симметричной связности, согласованной с метрикой, имеют вид

$$
R_{j k l ; m}^{i}+R^{i}{ }_{j m k ; l}+R^{i}{ }_{j l m ; k}=0 .
$$

Из (А.9) и свойств симметрии тензора кривизны следует, что

$$
R^{n}{ }_{i l m ; n}=R_{i m ; l}-R_{i l ; m}, \quad R_{m ; l}^{l}=\frac{1}{2} R_{; m} .
$$

Повторное дифференцирование (А.10) приводит к тождествам

$$
R_{; l n}^{i l k n}=R_{; l}^{i k}{ }_{; l}^{l}-R_{l i l ;}^{i l, \quad} R_{; l m}^{l m}=\frac{1}{2} R_{; m}{ }^{m} .
$$

Используя (А.10) и имеющее место для симметричной связности и произвольного тензора 2-го ранга тождество

$$
\left(\nabla_{l} \nabla_{k}-\nabla_{k} \nabla_{l}\right) A^{i l}=R_{n k l}^{i} A^{n l}-R_{n k} A^{i n},
$$

получим следуюшее следствие тождеств Бианки:

$$
R_{; k l}^{i l}=\frac{1}{2} R^{; i}{ }_{k}+R_{m k l}^{i} R^{m l}-R^{i m} R_{m k} .
$$




\section{ПРИЛОЖКНИЕ Б}

Здесь приведены выражения для некоторых геометрических величин в $N$-мерном однородном изотропном пространстве-времени с метрикой (28).

Символы Кристоффеля:

$$
\Gamma_{00}^{0}=\frac{a^{\prime}}{a} \equiv c, \quad \Gamma_{0 j}^{i}=c \delta_{j}^{i}, \quad \Gamma_{\alpha \beta}^{0}=c \gamma_{\alpha \beta}, \quad \Gamma_{\beta \delta}^{\alpha}\left(g_{i k}\right)=\Gamma_{\beta \delta}^{\alpha}\left(\gamma_{\nu \mu}\right) .
$$

Компоненты тензора Риччи и скалярная кривизна:

$$
\begin{aligned}
R_{00} & =(N-1) c^{\prime}, \quad R_{\alpha \beta}=-\gamma_{\alpha \beta}\left[c^{\prime}+(N-2)\left(c^{2}+K\right)\right], \\
R & =a^{-2}(N-1)\left[2 c^{\prime}+(N-2)\left(c^{2}+K\right)\right] .
\end{aligned}
$$

Компоненты тензора Эйнштейна:

$$
G_{00}=-\frac{(N-1)(N-2)}{2}\left(c^{2}+K\right), \quad G_{\alpha \beta}=\gamma_{\alpha \beta}(N-2)\left[c^{\prime}+\frac{(N-3)}{2}\left(c^{2}+K\right)\right] .
$$

Используя (Б.2), (Б.3) и формулу

$$
R_{l m p q} R^{l m p q}-4 R_{l m} R^{l m}+R^{2}=C_{l m p q} C^{l m p q}-\frac{4(N-3)}{N-2}\left(R_{l m} R^{l m}-\frac{N R^{2}}{4(N-1)}\right),
$$

в однородном изотропном случае получим

$$
\begin{aligned}
R_{l m p q} R^{l m p q}-4 R_{l m} R^{l m}+R^{2}= & a^{-4}(N-1)(N-2)(N-3)\left(c^{2}+K\right) \times \\
& \times\left[4 c^{\prime}+(N-4)\left(c^{2}+K\right)\right] .
\end{aligned}
$$

Компоненты ${ }^{(1)} H_{i k}$ и $^{(3)} H_{i k}$ выражаются следующими формулами:

$$
\begin{aligned}
{ }^{(1)} H_{00}= & \frac{(N-1)^{2}}{a^{2}}\left[2 c^{\prime 2}-4 c^{\prime \prime} c-4(N-4) c^{\prime} c^{2}-\frac{c^{4}}{2}(N-2)(N-10)-\right. \\
& \left.-K(N-2)\left(\frac{N-2}{2} K+(N-6) c^{2}\right)\right], \\
{ }^{(1)} H_{\alpha \beta}= & \gamma_{\alpha \beta} a^{-2}(N-1)\left\{4 c^{(3)}+4(2 N-9) c^{\prime \prime} c+2(3 N-11) c^{2}+\right. \\
& +6\left(N^{2}-10 N+20\right) c^{\prime} c^{2}+\frac{1}{2}(N-2)\left(N^{2}-15 N+50\right) c^{4}+ \\
& \left.+K(N-2)\left[(N-5)(N-6) c^{2}+2(N-6) c^{\prime}+\frac{1}{2}(N-2)(N-5) K\right]\right\}, \\
{ }^{(3)} H_{00}= & a^{-2} 2^{-1}(N-1)(N-2)(N-3)\left(c^{2}+K\right)^{2}, \\
{ }^{(3)} H_{\alpha \beta}= & -\gamma_{\alpha \beta} a^{-2} 2^{-1}(N-2)(N-3)\left[4 c^{\prime}\left(c^{2}+K\right)+(N-5)\left(c^{2}+K\right)^{2}\right] .
\end{aligned}
$$

Благодарности. Автор благодарит проф. А. А. Гриба и участников семинара лаборатории теоретической физики им. А. А. Фридмана за обсуждение работы. Работа поддержана Министерством образования РФ, грант Е02-3.1-198. 


\section{Список литературы}

[1] А. А. Гриб, С. Г. Мамаев, В. М. Мостепаненко. Вакуумные квантовые эффекты в сильных полях. М.: Энергоатомиздат, 1988.

[2] Н. Бирелл, П. Девис. Квантованные поля в искривленном пространстве-времени. М.: Мир, 1984.

[3] A. A. Grib, E. A. Poberii. Helv. Phys. Acta. 1995. V. 68. P. 380; K. С. Мамаева, Н. Н. Трунов. ТМФ. 2003. Т. 135. № 1. С. 82.

[4] А. Д. Линде. Физика элементарных частиц и инфляционная космология. М.: Наука, 1990.

[5] M. Bordag, J. Lindig, V. M. Mostepanenko, Yu. V. Pavlov. Int. J. Mod. Phys. D. 1997. V. 6. P. 449; V. B. Bezerra, V. M. Mostepanenko, C. Romero. Mod. Phys. Lett. A. 1997. V. 12. P. 145; S. Habib, C. Molina-París, E. Mottola. Phys. Rev. D. 2000. V. 61. P. 024010.

[6] R. M. Wald. Commun. Math. Phys. 1977. V. 54. P. 1.

[7] D. Lovelock. J. Math. Phys. 1971. V. 12. P. 498.

[8] B. Zwiebach. Phys. Lett. B. 1985. V. 156. P. 315.

[9] C. В. Кетов. Введение в квантовую теорию струн и суперструн. Новосибирск: Наука, 1990.

[10] Я. Б. Зельдович, А. А. Старобинский. ЖЭТФ. 1971. Т. 61. С. 2161.

[11] C. Lanczos. Ann. Math. 1938. V. 39. P. 842.

[12] Х. Цикон, Р. Фрезе, В. Кири, Б. Саймон. Операторы Шредингера с приложениями к квантовой механике и глобальной геометрии. М.: Мир, 1990.

[13] T. S. Bunch. J. Phys. A. 1979. V. 12. P. 517.

[14] S. A. Fulling. Aspects of Quantum Theory in Curved Space-Time. Cambridge: Cambridge Univ. Press, 1991.

[15] G. 't Hooft. Nucl. Phys. B. 1973. V. 61. P. 455.

[16] Yu. V. Pavlov. Int. J. Mod. Phys. A. 2002. V. 17. P. 1041.

[17] Ю. В. Павлов. ТМФ. 2004. Т. 138. № 3. С. 453.

[18] С. Г. Мамаев, В. М. Мостепаненко, В. А. Шелюто. ТМФ. 1985. Т. 63. № 1. С. 64.

[19] S. Mignemi, N. R. Stewart. Phys. Rev. D. 1993. V. 47. P. 5259.

[20] E. W. Mielke, F. E. Schunck. Nucl. Phys. B. 2000. V. 564. P. 185.

Поступила в редакцию 21.X.2003 г. 